\title{
Towards Automated Parameter Optimisation of Machinery by Persisting Expert Knowledge
}

\author{
Richard Nordsieck ${ }^{1}$, Michael Heider ${ }^{2}$, Andreas Angerer ${ }^{1}$ and Jörg Hähner ${ }^{2}$ \\ ${ }^{1}$ XITASO GmbH IT \& Software Solutions, Augsburg, Germany \\ ${ }^{2}$ Organic Computing Group, University of Augsburg, Augsburg, Germany
}

Keywords: Additive Manufacturing, Transfer Learning, Domain Adaption, Machine Learning, Knowledge Representation.

Abstract: Commissioning of machines takes up a considerable share of time and money of the total cost of developing a machine. Our project aims at developing an approach to decrease the time needed to commission machines by automating parameter optimisation with the help of formalised expert knowledge. The approach will be developed on the Fused Deposition Modelling (FDM) process, which is an additive manufacturing technique. We pay particular attention to keeping the approach sufficiently abstract to be applied to machines from other domains to benefit its industrial application.

\section{INTRODUCTION}

Commissioning of machinery can consume up to $25 \%$ of the total project duration according to a study of the German Machine Tool Builders' Association (VDW, 1997). They also state that up to $90 \%$ of this time is spent on commissioning the electronics and control software. As stated in (Abel, 2017), the commissioning process is done mainly by specialized workers, as it is a complex process and needs expert knowledge.

The geographic location of manufacturers of machinery and their customers is not necessarily identical. This leads to employment of highly-skilled professionals abroad, which is even more cost intensive due to increased management costs as well as travel and accommodation expenses. Therefore, commissioning is a prime target for cost and time optimisation of the manufacturing process overall, which is crucial for manufacturers to compete in a globalised economy. Furthermore, customers of manufacturing systems also have a strong interest in short commissioning times, as they are a prerequisite for expeditious use of newly acquired systems. Given the trend towards Reconfigurable Manufacturing Systems (Koren, 2006), shorter commissioning times gain even more importance: Every reconfiguration of a manufacturing system requires a subsequent commissioning to ensure proper operation of the machinery in changed configuration.

We differentiate between static and dynamic com- missioning. Static commissioning denotes the mechanical installation, operative checks of a machine or plant and, if needed, programming of control software. After static commissioning, single units and their integration can be successfully tested. This can usually be accomplished off-site e.g. at the manufacturer, at least for initial machine setup. Dynamic commissioning, however, describes the finetuning of process parameters until a satisfactory quality level of the produced part is achieved. In contrast to static commissioning, it can be seen as proof of a correctly working system under environmental influences. During dynamic commissioning, changes in the parametrisation of machinery as well as slight modifications to the control software might be necessary.

To reduce the duration of commissioning, the concept of virtual commissioning was introduced (Lee and Park, 2014; Hoffmann et al., 2010). In this concept, commissioning is simulated in varying levels of detail, which allows testing the interactions of components as well as the control programs, which are usually based on programmable logic controllers (PLCs)). This allows for early detection and remedying of faults, which account for a substantial amount of time spent during the commissioning procedure (VDW, 1997). Thereby, virtual commissioning is able to reduce the static part of the commissioning to the mechanical and electrical installation. However, one drawback of virtual commissioning is the 


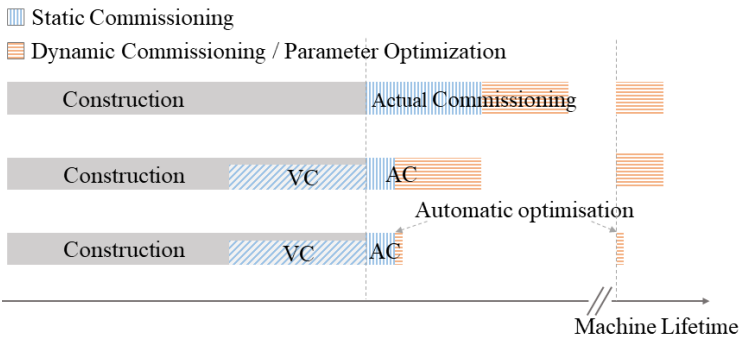

Figure 1: Schematic visualisation of machine lifetime. First the machine is constructed, which is followed by commissioning. Later on in the lifetime of a machine reparametrisation might be needed. While the top bar shows a traditional lifetime, the middle depicts a lifetime, which is optimised by the use of virtual commissioning (VC). The bottom bar shows the lifetime after automatic parameter optimisation.

effort required to create a digital twin of the plant or machine. While this may amortise quickly for larger manufacturing systems, plants or manufacturers that sell the same machine multiple times, manufacturers of special-purpose machinery find it hard to justify the recurring expenditure.

Nevertheless, the actual commissioning, which includes the dynamic commissioning, remains necessary as it is partly depending on environment-specific conditions (Abel, 2017). Thus, adjustments that compensate influences e.g. by temperature, humidity or raw material composition can only be performed onsite where the machine is operated. Note again that this can be necessary during initial on-site installation of machinery, but also after re-configurations due to changes in production processes or target products. Consequently, our approach aims at reducing the time required for the dynamic part of commissioning shown in orange horizontal lines in Figure 1 , that is parameter optimisation. It can be applied in conjunction with virtual commissioning, which reduces static parts of the commissioning process, or as a stand alone solution.

Expert knowledge required for parameter optimisation is traditionally acquired by working under the supervision of more experienced commissioners in a trainer-trainee fashion as well as commissioning a vast amount of machines. Due to demographic change and subsequent shifts in the workforce many experts will retire in the foreseeable future. Manufacturers are therefore posed with the challenge of how to preserve their knowledge and stay competitive. While commissioners might have documented commissioning procedures in varying detail and formats, few manufacturers have successfully worked towards a comprehensive knowledge base, according to our knowledge.

Similarly to other production processes, Fused
Deposition Modelling (FDM) requires experts to conduct parameter optimisations to produce parts of satisfactory quality. Due to the small form-factor of FDM machines, it is feasible to control environmental influences. Furthermore, parametrisations can be tested on multiple different parts without the need to manufacture tools, as is the case in other production processes. Therefore, we chose the FDM process to develop an approach to automatic parameter optimisation in a controlled environment. We see FDM as a representative of other production processes that depend on environmental conditions. After a successful verification of our approach on FDM, we study the transferability of the approach to other production processes, working towards our goal of decreasing their commissioning times by automating parameter optimisation.

This paper identifies challenges for automating parameter optimisation and outlines an approach on how to solve these. Section 2 provides a detailed view on production processes of additive manufacturing, based on which we will develop and verify our approach. Challenges for automating dynamic commissioning are introduced in Section 3. Our approach to automate parameter optimization by combining expert knowledge and machine learning is detailed in Section 4. Section 5 concludes this paper.

\section{CASE STUDY}

As described in Section 1, we decided to develop our approach on the Fused Deposition Modelling (FDM) process, which is an additive manufacturing technique, in which a nozzle lays down material layerwise until the desired object is constructed (Gibson et al., 2015). To facilitate this, the material is heated above its melting point and extruded through the nozzle. While extruding the nozzle moves along a preplanned path at a given speed. When the nozzle needs to move without extruding, e.g. when printing two non-connected models at once, the material is first retracted to decrease pressure in the extruder. Many printers use a heated bed on which the first layer is placed. The heating increases adhesion and slows down the effects of thermal expansion (or in this case contraction). We chose FDM since it is a controllable manufacturing process with multitudes of influence factors and configurable parameters. In addition, the results can be analysed by hand without the need of extensive training, a laboratory or other highly specialized equipment.

Additive manufacturing has become increasingly popular since its commercial introduction in the late 


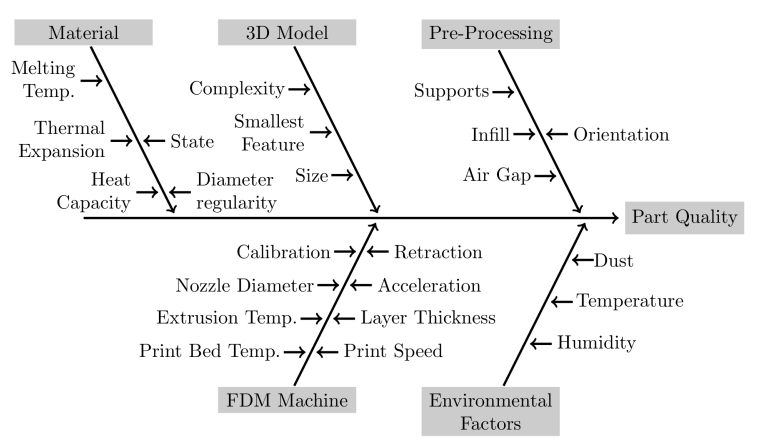

Figure 2: Cause and effect (Ishikawa) diagram of the for this work most important influence factors on part quality in an FDM production process.

1980s and early 1990s (Wohlers and Gornet, 2014). It is mostly used for rapid prototyping and manufacturing of small quantities of items, where moulding, casting and other conventional techniques would be too expensive. Another benefit of additive manufacturing is the ability to produce complex forms. These can be found in medical applications or if weight is of concern.

The main development in the scope of this project will be done on different cartesian and delta FDM printers using different plastics, such as PLA, ABS and PETG. After thoroughly evaluating our approach on multiple FDM printers, we will move on to different additive manufacturing techniques such as binder jetting (BJ) and selective laser sintering (SLS). BJ is a process, where powdered material is fused into form by applying a binding agent. SLS is another powder bed based process, where material is sintered locally and thereby fused into form. For each process expert knowledge will be collected and formalised separately. While certain expertise can be directly applied to the other additive manufacturing techniques, some knowledge is most likely very specific and differs between them. Thus, we will be able to test the transfer of knowledge between different machines of the same production process as well as between different additive manufacturing production processes. This marks the first step towards an abstract universal architecture.

The final quality of a 3D printed part is dependent on various factors with differing degrees of influence (Mohamed et al., 2015). Some factors can be quantified or measured better than others. The analysis of those factors can be done in discretionary detail. We have isolated the factors deemed most important in this context for the case of a generic FDM process in Figure 2. As we focus on developing an approach for parameters that are adjustable by software, some machine parameters, like nozzle diameter or filament width, can be seen as set. To offset the influences of wear and tear in mechanical parts on our machine learning systems, we plan on replacing the printers with new off the shelf versions during the project. We will focus on the commissioning and initial parametrisation of a system and thus not investigate machine degradation influences on optimal parameter selection.

There are several environmental factors that affect the quality of a produced part. In the following we will provide an overview of the environmental factors most relevant to FDM printing as well as to parameters used to mitigate their effects. Humidity has a significant impact on material quality. Due to the time it requires to infuse the filament it is more relevant during storage of filaments than during the printing process. Dust can, depending on amount and composition, decrease print quality and do serious damage to the machine by inflaming. Temperature is another relevant factor. On a process-agnostic perspective excessive heat or cold could do substantial damage to machine parts. On a process-related perspective the effects are more subtle. When molten plastics cool down they solidify and contract, the degree of which is dependent on the temperature and specific plastic. In the case of FDM printers this can lead to warping. Warping is a process, where segments of the printed part deform (curling upwards, away from the build plate, along the parts edges). It typically occurs as the lower-layers of the part cool down too fast. Since freshly extruded material needs to cool quicker than it would under room temperature $\left(21^{\circ} \mathrm{C}\right)$ most printers include a cooling fan to increase print quality. We will initially focus on temperature as a factor, as it, opposed to humidity, directly influences the process of FDM printing. Another relevant factor that often varies under real-word conditions is raw-material quality and composition, which influences print quality due to different temperature and form related properties. As such, we will conduct experiments, varying both temperature and raw-materials leading to a dataset, which we will use to train algorithmic approaches to parameter optimisation.

The main FDM parameters modifiable through software are either related to temperature, movement or the preprocessing (i.e. slicing, the generation of support structures and machine path planning). Printing bed and extrusion temperature can offset low surrounding temperatures while cooling fan speed can aid the cooling of freshly extruded filament. Layer thickness not only influences the optical appearance of a part but also the material properties during and after printing. Bridging is a part of the part construction process, where the printer tries to extrude material in an area, where no (immediate) lower layer is present, 
thus bridging two segments of lower layer together. Bridging becomes harder to accomplish if the part cools down too fast or too slow. Low speed can also result in bridges failing. Intelligent support placing could offset arising issues as bridges would become shorter. In the best case issues could also be prevented by simple print orientation changes, e.g. bottom up (Kozior and Kundera, 2017). Since we want to focus on parameters that are transferable to other production processes, we will focus on optimising parameters relating to the actual process as opposed to parameters changing the structure of the produced part. Vibrations that increase with velocity changes of the nozzle disrupt precise printing. Lowering the print speed and accelerations can accommodate for that (Alafaghani et al., 2017). Slower printing also tends to allow the extruded material to better set in place, rather than getting dragged along. Adjusting print speed and acceleration quickly becomes a trade-off between quantity and quality of produced parts. The analysis of our experimental results will help to quantify this tradeoff.

\section{CHALLENGES}

Our research hypothesis is that parameter optimisation has a significant effect on the quality of produced parts and thereby the amount of time required for commissioning. Consequently, automating parameter optimisation has the potential to effectively reduce cost and time spent on commissioning manufacturing systems. To confirm this hypothesis we plan to contrast times required for manual parameter optimisation with those resulting from the application of our optimisation algorithm. Actually developing a dependable approach for this task, however, poses several challenges as outlined in this chapter.

\subsection{Identification and Quantification of Parameters}

In Section 2 we introduced several environmental influences that can have a detrimental effect on the production process of the machine that is to be commissioned. Correctly identifying and quantifying them is a challenging task. This becomes especially evident if one considers diverse raw materials and their possibly differing qualities, e.g. manufacturer or even batch dependent.

Furthermore, we introduced process parameters that can be varied to mitigate the detrimental effect of aforementioned environmental parameters. To validate that these parameters are actually relevant to the production process and all relevant parameters are taken into consideration, we will conduct structured interviews with companies that employ additive manufacturing at an industrial scale and other experts in the field.

Section 2 is limited to processes encountered in the domain of additive manufacturing. Since we aim for an approach that can also be applied to other production processes we need to arrive at a domainindependent definition of commissioning. This definition can then be used to define similarities between production processes and their commissioning procedures. To arrive at this definition we will also conduct structured interviews over a broad range of producers of manufacturing systems or manufacturers.

\subsection{Data-related Challenges}

Commissioning in full, is usually executed before the handover of the manufacturing system. Parameter optimisation can also be required after a change in rawmaterial quality, a refit to produce different parts or extensive maintenance work. Compared to the overall runtime of a manufacturing system, this leads to very few opportunities to collect data. To generate data a simulation of the production process in question could be employed. However, many production processes are heavily influenced by advanced thermodynamics and material properties. Therefore, simulating them to the degree of detail that is needed requires large amounts of time and resources. Furthermore, the simulation would be limited to one production process and not easily adaptable to additional processes, which would render it uneconomic. A more promising solution to the data sparsity could be achieved by incorporating data collected by predictive quality approaches already employed on similar machines in the field. This would make it easier to empirically detect correlations between environmental influences and corresponding parameter configurations.

Automatically assessing the quality of chosen parameters via assessing the quality of the produced product is another data-related challenge. This would require the presence of automatic quality measurement systems, which is not commonplace. Therefore, our envisioned approach should actively request feedback of experts that manually assess the product's quality. 


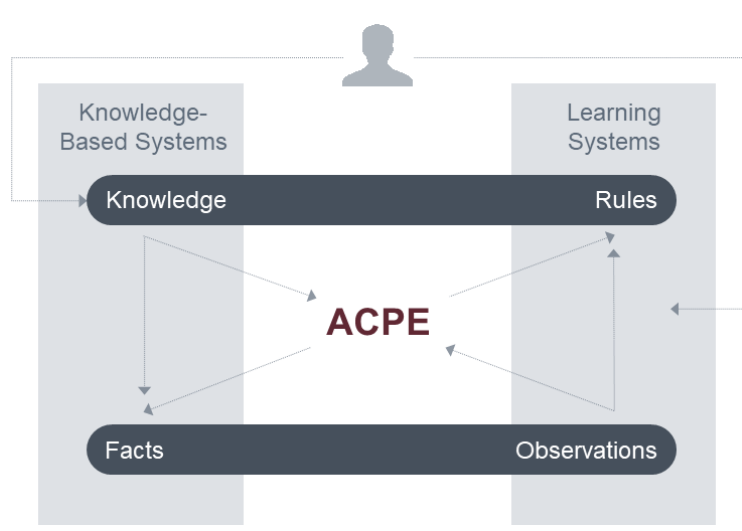

Figure 3: Schematic description of knowledge based (topdown) and learning systems (bottom-up). Our proposed approach (ACPE) combines aspects of both and is consequently shown in the middle.

\section{TOWARDS AUTOMATIC COMMISSIONING}

As outlined in Section 3, one of the main challenges for applying learning systems is the scarcity of commissionings of identical machines, which leads to few opportunities for collecting data. To mitigate the resulting uncertainties and training difficulties we propose an approach that combines learning and knowledge based methods. Based on a large amount of observations, learning systems generalize knowledge. As such, they work in a bottom-up manner. Contrary to that, knowledge based systems work as a top-down approach, inferring facts from a knowledge- and rulebase (Liao, 2005). A high level overview of these paradigms and their proposed interactions with our approach can be seen in Figure 3. This sections outlines our approach to achieve automatic parameter optimisation for commissioning under the constraint of sparse data and places it in academic context.

\subsection{Learning Quality Prediction and Strategy Selection}

In the context of FDM printing we see machine learning as a viable approach for achieving suitable quality prediction and strategy selection. In a reinforcement learning context a strategy would also be referred to as a policy. The ideal strategy would be to select process parameters (the action the system should take) for each set of 3D model, printer, environment and material (forming the state the system finds itself in) in a manner that facilitates the highest possible quality. Predicting the print quality requires an algorithm to predetermine the quality of a part produced by applying an action to a specific state. Quality prediction and strategy selection are generally different machine learning tasks, namely regression and planning. We will solve those with different base algorithms forming an actor-critic method (Sutton et al., 2000). Nonetheless, the results obtained by quality prediction can greatly improve strategy selection. Given an advanced quality prediction system each state-action pair can be evaluated on the fly without the need to real world test it by printing it, resulting in modelbased learning, which would be considerably faster. If the described ideal strategy was known, it would allow instantaneous commissioning of all printers for all tasks without the need of any commissioning time. An ideal partial strategy selects optimal actions (returning the highest quality part) for one given printer. We expect partial strategies to be related to each other and in conjunction to be able to closely describe the ideal strategy, if enough partial strategies are known.

Finding a quality prediction system, that is able to successfully generate reliable quality predictions for all state-action pairs, will not be achievable due to the large variety of printers and models. However we will create partial prediction systems that are able to perform for individual or small sets of printers, while trying to maintain an as large as possible variety of predictable models. Those partial prediction systems will be able to generalise for similar printers. The main challenge for constructing the prediction system will be the availability of data as there are-to our knowledge-no public comprehensive 3D printing data sets and as such all training data will have to be generated. To solve the quality prediction problem we will investigate the use of various classification techniques such as linear regression, decision trees (Kotsiantis, 2013), support vector machines and artificial neural networks. Depending on the algorithm we will either model classifications as individual regression problems or engineer a metric to transform class based assessments into a single variable description. This is done to ensure comparability. Beyond the algorithm itself we see two general approaches to the problem: To either try to directly incorporate the model as a whole into the classifier or to only use an abstract representation for it. The former would result in the use of learned convolutions (in the form of neural network layers) to extract core features and the latter would require an advanced (computable) metric to describe model complexity. During this project we will research the most feasible classification algorithm and approach for our problem as they both have advantages and drawbacks. Convolutional networks typically need numerous training samples, in- 
creasing with depth, until good results are achieved. When, like in this case, the exact targets are unknown, but rather represent a partial input for classification, learning the correct convolution becomes even harder. Yet they are able to learn complex connections for which finding an accurate algorithmic description would normally require large amounts of time.

To find reasonable strategies we plan to utilise Extended Classifier Systems (XCS) (Wilson, 1995; Butz and Wilson, 2002) a derivative of Learning Classifier Systems (LCS) (Holland, 1976). While standard XCS typically takes binary inputs we will encounter continuous (e.g. temperatures or speeds) or other non binary inputs and therefore need the ability to take real value inputs, which becomes possible in the XCS extension XCSR (Wilson, 2000). XCSR uses IF-THEN rules with an assigned quality (accuracy in predicting reward). Those IF-THEN rules are typically generated and optimised by a steady-state niche genetic algorithm (GA) but they can also be created by humans, for example by formalising the knowledge of a pool of experts as described in Section 4.2. The strategy itself will need to be able to extrapolate knowledge from known state-action pairs and combine it with existing knowledge. To fill existing knowledge gaps, as formerly defined by Stein et al. (Stein et al., 2018), active learning (Cohn et al., 1994) can be employed (Stein et al., 2017a). In addition we will expect to be able to use interpolation between known classifiers to gain knowledge on inter-laying classifiers (Stein et al., 2016; Stein et al., 2017b). Figure 4 illustrates the basic procedure. Known points (sets of parameters) in the multi-dimensional parameter space, that are similar to the parameter combination for which the quality is to be determined, are selected and interpolated to determine the new quality.

\subsection{Incorporating Expert Knowledge}

Historically, there have been multiple options of integrating expert knowledge with algorithms. The most prominent are expert systems. Expert systems consist of manually curated knowledge bases that contain facts and rules, as well as inference engines that deduce facts based on the associated knowledge bases (Liao, 2005). However, formalising expert knowledge to a sufficient degree is a complex task, especially since experts tend to take a certain amount of common-sense for granted, omitting crucial information about the process. Furthermore they can only dedicate a limited amount of time since they are constantly required to work on projects creating more direct revenue.

Embeddings of knowledge bases are successfully

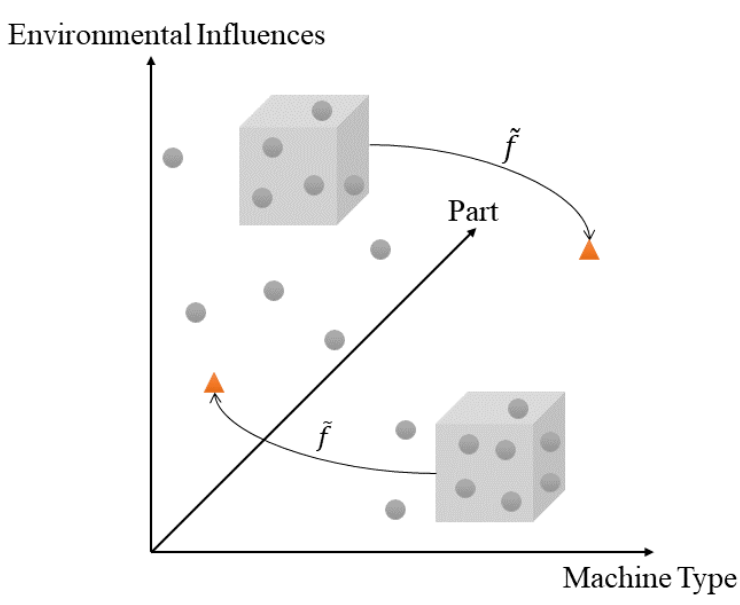

Figure 4: Illustration of a three dimensional subset of the parameter space, that includes the parameters, part to be produced (the pre-processed 3D Model) and machine type and the parameter group of environmental influences. Points for which the quality is known are displayed as grey circles. Points for which no knowledge is available are displayed as orange triangles. Using similarities between known and unknown points a set of known points (grey cube) is selected. A function $\tilde{f}$ allows interpolation of knowledge for the unknown point.

applied to increase performance of learning systems, for example in recommender systems (Wang et al., 2018; Sun et al., 2018) and text understanding (Wang et al., 2017; Yang and Mitchell, 2019). In the context of LCS Urbanowicz et al. experimented with the incorporation of expert knowledge for guiding the discovery components to explore attribute values found most predictive (Urbanowicz et al., 2012). In addition, reasoning tasks have been shown to fall short because of a lack of formalised common-sense knowledge being present in the data they have been trained on (Tandon et al., 2018). Similarly, there are approaches to mitigate this by incorporating formalisations of common-sense knowledge in the training process (Ma et al., 2018). Inspired by the successful combination of knowledge bases and learning systems outlined above, our approach seeks to mitigate the effect of scarce training data, by fusing of knowledge based and learning systems.

Knowledge about production processes, relevant parameters and influences is readily available in the industry. However, most of the time it is not documented in a structured and digitalized way. Therefore, we propose to work towards a knowledge base by persisting expert knowledge. As a first step we will form a semi-structure natural language knowledge base by recording expert knowledge of the FDM process with a markup language. This has the benefit of providing an easy to use interface for experts. Furthermore, if embedded in a documentation infrastructure, that 
other experts have access to, it provides a way of sharing knowledge throughout the company. While this infrastructure can already be considered a knowledge base, it is not yet easily computationally interpretable. Therefore, it is converted into a more formal representation consisting of conditions (Lehmann and Magidor, 1992) and triples, that describe relationships between entities (Nickel et al., 2016). Based on formal representations, multiple approaches exist in literature for knowledge base completion by automatically detecting and creating missing relations (Socher et al., 2013; Nguyen et al., 2018; Lin et al., 2015; Das et al., 2017). We will evaluate their applicability to processoriented knowledge bases. Lastly, these completed formal representations are then transformed into embeddings which can be incorporated into the learning algorithms to give them an understanding of process knowledge. We assume that this has a positive effect on the amount of data required for training.

As another approach addressing the scarcity of training data we want to evaluate transformations between knowledge representations. Knowledge in LCSs is represented by a learnt set of rules, whereas in ANNs it is represented by a matrix of neural weights. LCSs' knowledge representation is closer to that of a knowledge base, as in LCSs knowledge is modelled as IF-THEN rules (with an assigned quality). Thus, we will first evaluate a transformation between knowledge bases and LCSs before trying the same with ANNs.

\section{CONCLUSIONS}

This work illustrated the challenges faced during commissioning of machinery and detailed how automatic parameter optimisation can decrease both time needed for commissioning as well as down-times during the remainder of a machine's lifetime. We introduced FDM printing as a case study, outlining parameters that can be adjusted to increase its robustness against environmental factors. To achieve automatic parameter optimisation we outlined an approach to develop a learning system that could also facilitate on-boarding of and knowledge transfer between commissioners. A concrete next step will be the development of a pipeline to reproducibly conduct experiments with FDM printers to quantify the effect of different parameter configurations. In future research we will strive to realise the envisioned goal by implementing and evaluating the described approach on the dataset resulting from these experiments.

\section{ACKNOWLEDGEMENTS}

This work is supported by the German Federal Ministry for Economic Affairs and Energy (BMWi).

\section{REFERENCES}

Abel, M. (2017). Automatisierte Inbetriebnahme von rekonfigurierbaren Bearbeitungsmaschinen mit serviceorientierten Paradigmen. Stuttgart: Fraunhofer Verlag.

Alafaghani, A., Qattawi, A., Alrawi, B., and Guzman, A. (2017). Experimental Optimization of Fused Deposition Modelling Processing Parameters: A Design-forManufacturing Approach. Procedia Manufacturing, 10:791-803.

Butz, M. and Wilson, S. W. (2002). An Algorithmic Description of XCS. In Soft Comput. 6, pages 144-153.

Cohn, D., Ladner, R., and Waibel, A. (1994). Improving generalization with active learning. In Machine Learning, pages 201-221.

Das, R., Dhuliawala, S., Zaheer, M., Vilnis, L., Durugkar, I., Krishnamurthy, A., Smola, A., and McCallum, A. (2017). Go for a walk and arrive at the answer: Reasoning over paths in knowledge bases using reinforcement learning. arXiv preprint arXiv:1711.05851.

Gibson, I., Rosen, D., and Stucker, B. (2015). Additive Manufacturing Technologies - 3D Printing, Rapid Prototyping, and Direct Digital Manufacturing. Springer-Verlag.

Hoffmann, P., Schumann, R., Maksoud, T. M., and Premier, G. C. (2010). Virtual Commissioning Of Manufacturing Systems A Review And New Approaches For Simplification. In ECMS, pages 175-181.

Holland, J. H. (1976). Adaptation. In Progress in Theoretical Biology, volume 4, page 263-293. Academic Press, New York.

Koren, Y. (2006). General RMS Characteristics. Comparison with Dedicated and Flexible Systems. Reconfigurable Manufacturing Systems and Transformable Factories.

Kotsiantis, S. B. (2013). Decision trees: a recent overview. Artificial Intelligence Review, 39(4):261-283.

Kozior, T. and Kundera, C. (2017). Evaluation of the Influence of Parameters of FDM Technology on the Selected Mechanical Properties of Models. Procedia Engineering, 192:463-468.

Lee, C. G. and Park, S. C. (2014). Survey on the virtual commissioning of manufacturing systems. Journal of Computational Design and Engineering, 1(3):213222

Lehmann, D. and Magidor, M. (1992). What does a conditional knowledge base entail? Artificial intelligence, 55(1):1-60.

Liao, S.-H. (2005). Expert system methodologies and applications-a decade review from 1995 to 2004. Expert Systems with Applications, 28(1):93-103. 
Lin, Y., Liu, Z., Luan, H., Sun, M., Rao, S., and Liu, S. (2015). Modeling Relation Paths for Representation Learning of Knowledge Bases.

Ma, Y., Peng, H., and Cambria, E. (2018). Targeted aspectbased sentiment analysis via embedding commonsense knowledge into an attentive LSTM. In ThirtySecond AAAI Conference on Artificial Intelligence.

Mohamed, O. A., Masood, S. H., and Bhowmik, J. L. (2015). Optimization of fused deposition modeling process parameters: a review of current research and future prospects. Advances in Manufacturing, $3(1): 42-53$.

Nguyen, D. Q., Nguyen, T. D., Nguyen, D. Q., and Phung, D. (2018). A Novel Embedding Model for Knowledge Base Completion Based on Convolutional Neural Network. In Proceedings of the 2018 Conference of the North American Chapter of the Association for Computational Linguistics: Human Language Technologies, volume 2, pages 327-333.

Nickel, M., Murphy, K., Tresp, V., and Gabrilovich, E. (2016). A review of relational machine learning for knowledge graphs: From Multi-Relational Link Prediction to Automated Knowledge Graph Construction. Proceedings of the IEEE, 104(1):11-33.

Socher, R., Chen, D., Manning, C. D., and Ng, A. Y. (2013). Reasoning With Neural Tensor Networks for Knowledge Base Completion. In NIPS.

Stein, A., Maier, R., and Hähner, J. (2017a). Toward Curious Learning Classifier Systems: Combining XCS with Active Learning Concepts. In Proceedings of the Genetic and Evolutionary Computation Conference Companion, GECCO '17, pages 1349-1356, New York, NY, USA. ACM.

Stein, A., Rauh, D., Tomforde, S., and Hähner, J. (2016). Architecture of Computing Systems - ARCS 2016: 29th International Conference, Nuremberg, Germany, April 4-7, 2016, Proceedings, chapter Augmenting the Algorithmic Structure of XCS by Means of Interpolation, pages 348-360. Springer International Publishing, Cham.

Stein, A., Rauh, D., Tomforde, S., and Hähner, J. (2017b). Interpolation in the eXtended Classifier System: An Architectural Perspective. Journal of Systems Architecture, pages 79-94.

Stein, A., Tomforde, S., Diaconescu, A., Hähner, J., and Müller-Schloer, C. (2018). A Concept for Proactive Knowledge Construction in Self-Learning Autonomous Systems. In 2018 IEEE 3rd International Workshops on Foundations and Applications of Self* Systems $\left(F A S^{*} W\right)$, pages 204-213.

Sun, Z., Yang, J., Zhang, J., Bozzon, A., Huang, L.-K., and $\mathrm{Xu}, \mathrm{C}$. (2018). Recurrent knowledge graph embedding for effective recommendation. In Proceedings of the 12th ACM Conference on Recommender Systems, pages 297-305. ACM.

Sutton, R. S., McAllester, D. A., Singh, S. P., and Mansour, Y. (2000). Policy Gradient Methods for Reinforcement Learning with Function Approximation. In Solla, S. A., Leen, T. K., and Müller, K., editors, $A d$ vances in Neural Information Processing Systems 12, pages 1057-1063. MIT Press.
Tandon, N., Varde, A. S., and de Melo, G. (2018). Commonsense knowledge in machine intelligence. $A C M$ SIGMOD Record, 46(4):49-52.

Urbanowicz, R. J., Granizo-Mackenzie, D., and Moore, J. H. (2012). Using Expert Knowledge to Guide Covering and Mutation in a Michigan Style Learning Classifier System to Detect Epistasis and Heterogeneity, pages 266-275. Springer Berlin Heidelberg, Berlin, Heidelberg.

VDW (1997). Abteilungsübergreifende Projektierung komplexer Maschinen und Anlagen. VDW-Bericht.

Wang, H., Zhang, F., Xie, X., and Guo, M. (2018). Dkn: Deep knowledge-aware network for news recommendation. In Proceedings of the 2018 World Wide Web Conference on World Wide Web, pages 1835-1844. International World Wide Web Conferences Steering Committee.

Wang, J., Wang, Z., Zhang, D., and Yan, J. (2017). Combining Knowledge with Deep Convolutional Neural Networks for Short Text Classification. In IJCAI, pages 2915-2921.

Wilson, S. W. (1995). Classifier Fitness Based on Accuracy. In Evol. Comput. 3, page 149-175.

Wilson, S. W. (2000). Get Real! XCS with ContinuousValued Inputs. In Learning Classifier Systems, page 209-219. Springer Berlin Heidelberg,.

Wohlers, T. and Gornet, T. (2014). History of additive manufacturing. Wohlers report, 24(2014):118

Yang, B. and Mitchell, T. (2019). Leveraging knowledge bases in LSTMs for improving machine reading. arXiv preprint arXiv:1902.09091. 